Research Article

\title{
On a Discrete-Time Risk Model with Random Income and a Constant Dividend Barrier
}

\author{
Zhenhua Bao $\mathbb{D}$, Junqing Huang, and Jing Wang $\mathbb{D}$ \\ School of Mathematics, Liaoning Normal University, Dalian 116029, China \\ Correspondence should be addressed to Jing Wang; wjlnnu@126.com
}

Received 30 January 2021; Accepted 11 May 2021; Published 25 May 2021

Academic Editor: Xuewen Lu

Copyright (C) 2021 Zhenhua Bao et al. This is an open access article distributed under the Creative Commons Attribution License, which permits unrestricted use, distribution, and reproduction in any medium, provided the original work is properly cited.

In this paper, a discrete-time risk model with random income and a constant dividend barrier is considered. Under such a dividend policy, once the insurer's reserve hits the level $b(b>0)$, the excess of the reserve over $b$ is paid off as dividends. We derive a homogeneous difference equation for the expected present value of dividend payments. Corresponding solution procedures for the difference equation are invested. Finally, we give a numerical example to illustrate the applicability of the results obtained.

\section{Introduction}

In the actuarial literature, many authors focus their research interests on discrete-time risk models, which can be used as an approximation to continuous time models. Li et al. [1] present a review of results for discrete-time risk models, including the compound binomial risk model and some of its extensions. In recent years, discrete-time risk models with dependent structure have received increasing attention, and the readers are referred to Liu and Bao [2] for the related studies on different kinds of dependent models.

Additionally, problems related to dividends have been considered extensively in the discrete-time setting. Because of the certainty of ruin for a risk model with a constant dividend barrier, the calculation of the expected discounted dividend payment is a major problem of interest in the context. Among the class of discrete-time risk models, Tan and Yang [3] derive a recursive algorithm to compute a particular class of Gerber-Shiu penalty functions in the framework of the compound binomial model with randomized dividend payments. Landriault [4] then generalize Tan and Yang's model to consider the compound binomial model with a multithreshold dividend structure and randomized dividend payments. For the compound binomial risk model with possible delay of claims, Xie and Zou [5] investigate the expected present value of total dividends under stochastic interest rates. $\mathrm{Wu}$ and $\mathrm{Li}$ [6] further consider the discrete-time risk model with time-delayed claims and a constant dividend barrier, but the main claim is dependent on its associated by-claim. In the framework of a discrete semi-Markov risk model, a randomized dividend policy is studied by Yuen et al. [7]. Zhang and Liu [8] consider a discrete-time risk model with a mathematically tractable dependence structure between interclaim time and claim size in the presence of an impulsive dividend strategy.

All the risk models discussed above are based on a common assumption that the premium is collected with a positive deterministic constant. However, this assumption can be unrealistic and inappropriate in practical contexts because the insurance company may have lump sums of income. Therefore, many authors consider the risk models with stochastic income to capture the uncertainty of the customers' arrivals, for example, Boikov [9], Yang and Zhang [10], Labbé and Sendova [11], Karnaukh [12], Bao and Liu [13], and Zhou et al. [14].

In the present paper, we propose a discrete-time risk model with random income and a constant dividend barrier. A similar model has been discussed by Zhou et al. [14]. However, we analyze the model with a general premium rate $c$, while Zhou et al. [14] investigate the model with timedelayed claims and unit premium assumption. As stated in Landriault [4], unlike the classical compound Poisson risk 
model for which a unit premium rate can be assumed without loss of generality, such reasoning does not hold for the discrete-time risk model. On the contrary, the technique used in the present paper is to solve a high-order difference equation that often arises in the discrete-time risk model with general premium $c$.

The rest of the paper is organized as follows. A brief description of the discrete-time model and the introduction of the expected present value of total dividends are considered in Section 2. In Section 3, we obtain and solve a homogeneous difference equation satisfied by the expected present value of dividend payments. When the claim sizes follow the geometrical distribution, a closed-form solution to the expected present value of dividends and the corresponding numerical example are provided in Section 4. Finally, we end our works with conclusion in Section 5.

\section{The Model}

Throughout, denote by $\mathbb{N}$ the set of nature numbers and $\mathbb{N}^{+}=\mathbb{N} \backslash\{0\}$. Similar to Bao and Liu [13], we assume that the premium income is a binomial process $\left\{M_{k}, k \in \mathbb{N}\right\}$ with parameter $p_{0}\left(0<p_{0} \leq 1\right)$. More precisely, we denote by $p_{0}$, the probability that a premium of size $c\left(c \in \mathbb{N}^{+}\right)$is received, and $q_{0}=1-p_{0}$, the probability that no premium is received in each period. The number of claims is assumed to be another binomial process $\left\{N_{k}, k \in \mathbb{N}\right\}$ with parameter $p(0<p<1)$. It means that the probability of having a claim is $p$ and the probability of no claim is $q=1-p$ in each period. The claims $\left\{X_{j}, k \in \mathbb{N}^{+}\right\}$are independent and identically distributed (i.i.d.) positive and integer-valued random variables with the same distribution as the generic random variable $X$. The probability mass function (p.m.f.) and mean of $X$ are denoted by $f_{X}(x)$ and $\mu$, respectively. Let $S_{k}=\prod_{i=1}^{N_{k}} X_{i}$ be the total amount of settled claims up to the end of the $k$ th time period with $S_{0}=0$. The independence among $\left\{N_{k}, k \in \mathbb{N}\right\},\left\{M_{k}, k \in \mathbb{N}\right\}$, and $\left\{X_{j}, k \in \mathbb{N}^{+}\right\}$are also assumed.

Suppose that premiums are received at the beginning of each period, and claims are paid out at the end of each period. We introduce a dividend policy to the company that a certain amount of dividends will be paid to the policyholder instantly, as long as the surplus of the company at time $k$ is higher than a constant dividend barrier $b(b>0)$. It implies that the dividend payments will only possibly occur at the beginning of each period, right after receiving the premium payment. Then, the corresponding surplus $U_{b}(k)$ of the insurer at the end of the $k$ th time period can be described as

$$
U_{b}(k)=u+c M_{k}-S_{k}-D(k), \quad u=0,1,2, \ldots, b,
$$

where $U_{b}(0)=u$ is the initial surplus and $D(k)=D_{1}+D_{2}+$ $\cdots+D_{k}$ is the sum of the total dividend payments in the first $k$ period with $D(0)=0$. The amount of dividends $D_{k}$ paid out in the $k$ th time period is defined as

$$
D_{k}=\max \left\{U_{b}(k-1)+c \eta_{k}-b, 0\right\}, \quad k \in \mathbb{N}^{+},
$$

where $\left\{\eta_{k}, k \in \mathbb{N}^{+}\right\}$is a sequence of i.i.d. Bernoulli random variables with $\mathbb{P}\left(\eta_{k}=1\right)=p_{0}$ and $\mathbb{P}\left(\eta_{k}=0\right)=q_{0}$.

Note that the positive safety loading condition holds if $c p_{0}>p \mu$. The time of ultimate ruin for model (1) is defined as $\tau_{b}=\min \left\{k, U_{b}(k)<0\right\}$. Let $v(0<v \leq 1)$ be a constant annual discount rate for each period. Then, the expected present value of the dividend payments due till ruin is

$$
V(u ; b)=\mathbb{E}\left[\sum_{k=1}^{\tau_{b}}\left(D_{k}\right) v^{k-1} \mid U(0)=u\right] .
$$

\section{Expected Present Value of Dividend Payments}

By considering the occurrence (or not) of the premium income and claims in the next period, we separate the four possible cases as follows: no premium arrival and no claim occurs, a random premium arrival and no claim occurs, a random premium arrival and a claim occurs, and no premium arrival and a claim occurs. For $u=0,1, \ldots, b-c$, we can obtain the following result by using the total probability formula:

$$
\begin{aligned}
V(u ; b) & =v q_{0} q V(u ; b)+v p_{0} q V(u+c ; b)+v p_{0} p \sum_{x \leq u+c} V(u+c-x ; b) f(x)+v q_{0} p \sum_{x=1}^{u} V(u-x ; b) f(x) \\
& =v q_{0} q V(u ; b)+v p_{0} q V(u+c ; b)+v p_{0} p(V * f)(u+c)+v q_{0} p(V * f)(u),
\end{aligned}
$$

where $V * f$ holds for the convolution product of $V$ and $f$.

Now, we show that (4) is a homogeneous difference equation of order $c$. The (forward) difference operator $\Delta$ is defined as

$$
\Delta V(u ; b)=V(u+1 ; b)-V(u ; b) .
$$

Using the property of the forward difference operator $\Delta$, we obtain

$$
V(u+c ; b)=\sum_{j=0}^{c}\left(\begin{array}{l}
c \\
j
\end{array}\right) \Delta^{j} V(u ; b) .
$$




$$
V(u ; b)=v q_{0} q V(u ; b)+v p_{0} q \sum_{j=0}^{c}\left(\begin{array}{l}
c \\
j
\end{array}\right) \Delta^{j} V(u ; b)+v p_{0} p \sum_{j=0}^{c}\left(\begin{array}{l}
c \\
j
\end{array}\right) \Delta^{j}(V * f)(u)+v q_{0} p(V * f)(u),
$$

or, equivalently

$$
\sum_{j=0}^{c} a_{j}\left(\begin{array}{c}
c \\
j
\end{array}\right) \Delta^{j} V(u ; b)=\sum_{j=0}^{c} b_{j}\left(\begin{array}{c}
c \\
j
\end{array}\right) \Delta^{j}(V * f)(u)
$$

where

$$
\begin{aligned}
& a_{j}=1_{\{j=0\}}-v q_{0} q 1_{\{j=0\}}-v p_{0} q, \\
& b_{j}=v q_{0} p 1_{\{j=0\}}+v p_{0} p .
\end{aligned}
$$

Letting $A(z)=\sum_{j=0}^{c} a_{j} z^{j}$ and $B(z)=\sum_{j=0}^{c} b_{j} z^{j}$, which are two polynomials of degree $c$ in $z$. Then, (8) can be reformulated as

$$
A(\Delta) V(u ; b)=B(\Delta)(V * f)(u) .
$$

Equation (10) implies that $V(u ; b)$ satisfies a homogeneous difference equation of order $c$. From the general theory of difference equations, the solution to (10) can be expressed as

$$
V(u ; b)=\sum_{j=0}^{c-1} \alpha_{j} y_{j}(u ; b),
$$
where $\left\{y_{j}(u ; b)\right\}_{u=0}^{\infty}$ are linearly independent and satisfy

$$
A(\Delta) y(u ; b)=B(\Delta)(y * f)(u) .
$$

Multiplying (12) by $z^{u+c}$ and then summing over $u$ from 0 to $\infty$ lead to

$$
\sum_{u=0}^{\infty} z^{u+c} y(u ; b)=v q_{0} q \sum_{u=0}^{\infty} z^{u+c} y(u ; b)+v p_{0} q \sum_{u=0}^{\infty} z^{u+c} y(u+c ; b)+v p_{0} p \sum_{u=0}^{\infty} z^{u+c}(y * f)(u+c)+v q_{0} p \sum_{u=0}^{\infty} z^{u+c}(y * f)(u)
$$

which can be reformulated as

$$
\tilde{y}(z ; b)=\frac{-v p_{0}\left\{q \sum_{u=0}^{c-1} z^{u} y(u ; b)+p \sum_{u=0}^{c-1} z^{u}(y * f)(u)\right\}}{\left(1-v q_{0} q\right) z^{c}-v\left(p_{0} q+p \tilde{g}(z) \tilde{f}(z)\right)}
$$

To find a set of fundamental solutions to (14), we choose $y_{j}(u ; b)=1_{\{j=u\}}$ for $j, u \in\{0,1, \ldots, c-1\}$. An application of Theorem 3.4 in [15] implies that $\left\{y_{j}(u ; b)\right\}_{u=0}^{\infty}$, for $j=0,1, \ldots, c-1$, are linearly independent. From (14), the generating functions associated with $\left\{y_{j}(u ; b)\right\}_{u=0}^{\infty}$ satisfy

$$
\tilde{y}_{j}(z ; b)=\frac{-R_{j}(z)}{\widetilde{h}_{1}(z)-\widetilde{h}_{2}(z)},
$$

where

$$
\begin{aligned}
\tilde{h}_{1}(z) & =\left(1-v q_{0} q\right) z^{c}, \\
\widetilde{h}_{2}(z) & =v\left(p_{0} q+p \tilde{g}(z) \tilde{f}(z)\right), \\
\tilde{g}(z) & =: \sum_{k=0}^{\infty} g(z) z^{k}=p_{0}+q_{0} z^{c}, \\
R_{j}(z) & =v p_{0}\left\{q z^{j}+p \sum_{u=j+1}^{c-1} z^{u} f(u-j)\right\} .
\end{aligned}
$$

We can easily identify the number of zeros on the denominator in (15) by using Rouché's theorem and its generalization. The proof is omitted, and similar discussions can be found in Bao and Liu [16].
Lemma 1. When $0<v<1$, the denominator in (15) has exactly $c$ solutions, say $\left\{z_{i}\right\}_{i=1}^{c}$, inside the unit circle $\mathscr{C}=\{z:|z|=1\}$.

Lemma 2. When $v=1$, the denominator in (15) has exactly $c-1$ solutions, say $\left\{z_{i}\right\}_{i=1}^{c-1}$, inside the unit circle $\mathscr{C}=\{z:|z|=1\}$ and another trivial root $z_{c}=1$.

In what follows, we assume these zeros $\left\{z_{i}\right\}_{i=1}^{c}$ are distinct for simplicity, as the analysis of the multiple $z_{i}$ 's leads to tedious derivations. We are ready to give alternative expressions for both the numerator and the denominator in (15) with the help of $\left\{z_{i}\right\}_{i=1}^{c}$. Since the numerator in (15) is analytic for all $\operatorname{Re}(z) \in[-1,1]$, the zeros of the denominator in (15) have to be zeros of the numerator.

Let $\pi_{c}(z)=\prod_{j=1}^{c}\left(z-z_{j}\right)$ and $\pi_{c}^{\prime}\left(z_{k}\right)=\prod_{j=1, j \neq k}^{c}\left(z_{k}\right.$ $-z_{j}$ ). Since $\widetilde{h}_{1}(z)$ is a polynomial of degree $c$ in $z$, using the Lagrange interpolating theorem, one knows

$$
\widetilde{h}_{1}(z)=\pi_{c}(z)\left(\frac{\widetilde{h}_{1}(0)}{\pi_{c}(0)}+\sum_{i=1}^{c} \frac{\tilde{h}_{2}\left(z_{i}\right)}{z_{i}} \frac{1}{\pi_{c}^{\prime}\left(z_{i}\right)}+\sum_{i=1}^{c} \frac{\widetilde{h}_{2}\left(z_{i}\right)}{z-z_{i}} \frac{1}{\pi_{c}^{\prime}\left(z_{i}\right)}\right) .
$$

It is easy to see that $\lim _{z \longrightarrow \infty} \widetilde{h}_{1}(z) / \pi_{c}(z)=1-v q_{0} q$, and (17) can be simplified as

$$
\frac{\widetilde{h}_{1}(z)}{\pi_{c}(z)}=1-v q_{0} q+\sum_{i=1}^{c} \frac{\widetilde{h}_{2}\left(z_{i}\right)}{z-z_{i}} \frac{1}{\pi_{c}^{\prime}\left(z_{i}\right)},
$$

which yields 


$$
\frac{\widetilde{h}_{1}(z)-\widetilde{h}_{2}(z)}{\pi_{c}(z)}=1-v q_{0} q-\left(\frac{\widetilde{h}_{2}(z)}{\pi_{c}(z)}-\sum_{i=1}^{c} \frac{\widetilde{h}_{2}\left(z_{i}\right)}{z-z_{i}} \frac{1}{\pi_{c}^{\prime}\left(z_{i}\right)}\right) .
$$

Now, we use a discrete operator $\mathscr{T}_{z}$ defined as $\mathscr{T}_{z} y(c)=\sum_{u=0}^{\infty} z^{u} y(u+c)$, and the corresponding properties of $\mathscr{T}_{z}$ can be found in Li [17]. Then, $h_{2}(z)$ can be rewritten as

$$
\widetilde{h}_{2}(z)=v p z^{c} \mathscr{T}_{z}(g * f)(c)+\eta(z)
$$

where $\eta(z)=v p_{0} q+v p \sum_{u=0}^{c-1} z^{u}(g * f)(u)$ is a polynomial of degree $c-1$ in $z$. By imitating the same steps discussed above, we can prove that

$$
\frac{\eta(z)}{\pi_{c}(z)}-\sum_{i=1}^{c} \frac{\eta\left(z_{i}\right)}{z-z_{i}} \frac{1}{\pi_{c}^{\prime}\left(z_{i}\right)}=0 .
$$

Therefore, substituting (20) into (19) yields

$$
\begin{aligned}
\frac{\widetilde{h}_{1}(z)-\widetilde{h}_{2}(z)}{\pi_{c}(z)} & =1-v q_{0} q-v p\left(\frac{z^{c} \mathscr{T}_{z}(g * f)(c)}{\pi_{c}(z)}-\sum_{i=1}^{c} \frac{\left(z_{i}\right)^{c} \mathscr{T}_{z_{i}}(g * f)(c)}{z-z_{i}} \frac{1}{\pi_{c}^{\prime}\left(z_{i}\right)}\right) \\
& =1-v q_{0} q-v p \mathscr{T}_{z} \mathscr{T}_{z_{c}} \ldots \mathscr{T}_{z_{2}} \mathscr{T}_{z_{1}}(g * f)(c) .
\end{aligned}
$$

Note that (15) can be modified as

$$
\tilde{y}_{j}(z ; b)=\frac{-R_{j}(z) / \pi_{c}(z)}{\left(\widetilde{h}_{1}(z)-\widetilde{h}_{2}(z)\right) / \pi_{c}(z)} .
$$

$$
\frac{-R_{j}(z)}{\pi_{c}(z)}=\sum_{k=1}^{c} \frac{R_{j}\left(z_{k}\right)}{\pi_{c}^{\prime}\left(z_{k}\right)} \frac{1}{z_{k}-z} .
$$

By inserting (22) and (24) into (23), we obtain

For the numerator in (23), partial fractions lead to an equivalent representation

$$
\tilde{y}_{j}(z ; b)=\frac{1}{1-v q_{0} q}\left[v p \tilde{y}_{j}(z ; b) \mathscr{T}_{z} \mathscr{T}_{z_{c}} \ldots \mathscr{T}_{z_{2}} \mathscr{T}_{z_{1}}(g * f)(c)+\sum_{k=1}^{c} \frac{R_{j}\left(z_{k}\right)}{\pi_{c}^{\prime}\left(z_{k}\right)} \frac{1}{z_{k}-z}\right] .
$$

The direct inversion of the generating functions in (25) gives the following theorem immediately.

Theorem 1. For $j=0,1, \ldots, c-1, y_{j}(u, b)$ satisfies the following defective renewal equation:

$$
y_{j}(u ; b)=\varsigma \sum_{n=0}^{u} y_{j}(u-n ; b) \chi(n)+\zeta(u), \quad u \in \mathbb{N},
$$

where

$$
\begin{aligned}
\zeta(u) & =\frac{1}{1-v q_{0} q} \sum_{k=1}^{c} \frac{R_{j}\left(z_{k}\right)}{\pi_{c}^{\prime}\left(z_{k}\right)}\left(\frac{1}{z_{k}}\right)^{u+1}, \\
\varsigma & =\frac{v p}{1-v q_{0} q} \mathscr{T}_{1} \mathscr{T}_{z_{c}} \ldots \mathscr{T}_{z_{2}} \mathscr{T}_{z_{1}}(g * f)(c), \\
\chi(n) & =\frac{\mathscr{T}_{z_{c}} \ldots \mathscr{T}_{z_{2}} \mathscr{T}_{z_{1}}(g * f)(c+n)}{\mathscr{T}_{1} \mathscr{T}_{z_{c}} \ldots \mathscr{T}_{z_{2}} \mathscr{T}_{z_{1}}(g * f)(c)} .
\end{aligned}
$$

Proof. To complete the proof, it remains to show that $\varsigma<1$. In the case of $v \in(0,1)$, it follows from (22) that $\varsigma=\frac{1}{1-v q_{0} q}\left(1-v q_{0} q-\frac{\widetilde{h}_{1}(1)-\widetilde{h}_{2}(1)}{\pi_{c}(1)}\right)=1-\frac{1-v}{\left(1-v q_{0} q\right) \pi_{c}(1)}<1$.

In the case of $v=1$, since $z_{c}$ is a root of the denominator in (15), we have

$$
\widetilde{h}_{1}\left(z_{c}\right)-\widetilde{h}_{2}\left(z_{c}\right)=0 .
$$

Differentiating (29) with respect to $v$ and taking the limit $v \longrightarrow 1^{-}$, from the positive safety loading condition, we eventually find

$$
\lim _{v \longrightarrow 1^{-}} z_{c}^{\prime}=\frac{1}{c p_{0}-p \mu}>0 .
$$

Thus, taking the limit $v \longrightarrow 1^{-}$in (28), one deduces

$$
\lim _{v \longrightarrow 1^{-}} \varsigma=1-\frac{1}{\left(1-q_{0} q\right) \pi_{c}^{\prime}(1)} \lim _{v \longrightarrow 1^{-}} \frac{1-v}{1-z_{c}}<1,
$$

which completes the proof.

Starting from $y_{j}(u ; b)=1_{\{j=u\}}$ for $u=0,1, \ldots, b-c$, (26) allows for a recursive calculation of the fundamental solutions to the homogeneous difference (14). Regarding the constants $\alpha_{j}(j=0,1, \ldots, c-1)$ in the structural form of of 
(12), they are determined such that the remaining $c$ equations are satisfied with initial surplus $u=b-c+1, \ldots, b$, namely,

$$
\begin{aligned}
V(u ; b)= & v q_{0} q V(u ; b)+v p_{0} q(u+c-b+V(b ; b)) \\
& +v p_{0} p\left\{1_{\{x \leq u+c-b\}} f(x)[(u+c-b-x)+V(b ; b)]+\sum_{x=u+c-b}^{u+c} V(u+c-x ; b) f(x)\right\} \\
& +v q_{0} p \sum_{x=1}^{u} V(u-x ; b) f(x) .
\end{aligned}
$$

\section{Numerical Example}

In this section, we explain the solution procedure to the difference equation $V(u ; b)$ through a concrete example. In what follows, it is assumed that $f$ is a geometrical distribution with $f(n)=(1-\theta) \theta^{n-1}, n \in \mathbb{N}^{+}$, and the corresponding probability generating function is $\widetilde{f}(z)=((1-\theta) z / 1-\theta z)$. Thus, equation (15) becomes

$$
\tilde{y}_{j}(z ; b)=\frac{-R_{j}(z)(1-\theta z)}{\Lambda(z)},
$$

where $\Lambda \quad(z)=z^{c} \quad(1-\theta z)-v q_{0} q z^{c} \quad(1-\theta z)-$ $v p_{0} q(1-\theta z)-v p_{0} p(1-\theta) z-v q_{0} p(1-\theta) z^{c+1}$. Since $\Lambda(z)$ is a polynomial of degree $c+1$ with the leading coefficient $a=-\theta+v q_{0} q \theta-v q_{0} p(1-\theta)$, it can be factored as

$$
\Lambda(z)=a \pi_{c}(z)(z-\xi)
$$

where $\xi$ is the zero of $\Lambda(z)$ on the complex plane. Note that $\xi$ has a module larger than 1 and $z_{i}(i=1,2, \ldots, c)$ has a module less than 1. Moreover, routine calculations lead to

$$
\frac{\pi_{c}(z)(1-\theta z)}{\Lambda(z)}=\frac{\pi_{c}(z)(1-\theta z)}{a \pi_{c}(z)(z-\xi)}=-\frac{\theta}{a}\left(1+\frac{(1 / \theta)-\xi}{\xi-z}\right) .
$$

By substituting (24) and (35) into (33), we obtain

$$
\tilde{y}_{j}(z ; b)=\frac{(1-\theta z)}{\Lambda(z)} \sum_{k=1}^{c} \frac{R_{j}\left(z_{k}\right)}{\pi_{c}^{\prime}\left(z_{k}\right)} \frac{\pi_{c}(z)}{z_{k}-z}=-\frac{\theta}{a} \sum_{k=1}^{c} \frac{R_{j}\left(z_{k}\right)}{\pi_{c}^{\prime}\left(z_{k}\right)}\left(1+\frac{(1 / \theta)-\xi}{\xi-z}\right) \frac{1}{z_{k}-z} .
$$

Upon inversion, we obtain from (36) that

$$
\begin{aligned}
y_{j}(u ; b) & =-\frac{\theta}{a} \sum_{k=1}^{c} \frac{R_{j}\left(z_{k}\right)}{\pi_{c}^{\prime}\left(z_{k}\right)}\left[z_{k}^{-(u+1)}+\left(\frac{1}{\theta}-\xi\right) \sum_{l=0}^{u} \xi^{-(u+1-l)} z_{k}^{-(l+1)}\right] \\
& =-\frac{\theta}{a} \sum_{k=1}^{c} \frac{R_{j}\left(z_{k}\right)}{\pi_{c}^{\prime}\left(z_{k}\right)}\left(1-\frac{(1 / \theta)-\xi}{z_{k}-\xi}\right) z_{k}^{-(u+1)}-\frac{\theta}{a} \sum_{k=1}^{c} \frac{R_{j}\left(z_{k}\right)}{\pi_{c}^{\prime}\left(z_{k}\right)} \cdot \frac{(1 / \theta)-\xi}{z_{k}-\xi} \xi^{-(u+1)},
\end{aligned}
$$

where $R_{j}\left(z_{k}\right)$ is calculated through

$$
R_{j}(z)=v p_{0}\left\{q z^{j}+\frac{p(1-\theta) z^{j+1}}{1-\theta z}\left[1-(\theta z)^{c-j-1}\right] 1_{\{c-1 \geq j+1\}}\right\}
$$

As the explicit expression for $y_{j}(u ; b)$ is given by (37), the constants $\left\{\alpha_{j}\right\}_{j=0}^{c-1}$ can be achieved such that (32) holds for $u=b-c+1, \ldots, b$, which completes the determination of $V(u ; b)$. We provide a numerical example to illustrate the theoretical results.
Example 1. Suppose $c=2$, and from (33), we have

$$
\begin{aligned}
\Lambda(z)= & z^{2}(1-\theta z)-v q_{0} q z^{2}(1-\theta z)-v p_{0} q(1-\theta z) \\
& -v p_{0} p(1-\theta) z-v q_{0} p(1-\theta) z^{3} .
\end{aligned}
$$

Let $p=0.6, p_{0}=0.7$, and $v=0.85$, and the relative safety loading condition $c p_{0}>p \mu$ holds for all $\theta \in(0,4 / 7)$. By solving $\Lambda(z)=0$, we obtain the values of $z_{i}$ 's and $\xi$, see Table 1.

Explicit expressions for $y_{j}(u ; b)$ are determined by (37). For example, one has, for $\theta=0.3$, 
TABLE 1: Numerical results of $z_{i}$ and $\xi$ for $p=0.6, p_{0}=0.7, \theta=0.3$, and $v=0.85$.

\begin{tabular}{lccc}
\hline & $\xi$ & $z_{1}$ & $z_{2}$ \\
\hline$\theta=0.1$ & 3.48604534795459 & -0.363766275505389 & 0.824973674803551 \\
$\theta=0.2$ & 2.54139402542121 & -0.381251310060523 & 0.813367218414144 \\
$\theta=0.3$ & 1.98618491341006 & -0.39890721086863 & 0.797848459477162 \\
\hline
\end{tabular}

TABLe 2: Numerical results of $y_{j}(u ; b)$ for $b=15$.

\begin{tabular}{|c|c|c|c|c|}
\hline$u$ & 0 & 1 & 2 & 3 \\
\hline$y_{0}(u ; b)$ & 1 & $-5.52900 \times 10^{-17}$ & 3.45811 & -4.17551 \\
\hline$y_{1}(u ; b)$ & $3.31740 \times 10^{-17}$ & 1 & -1.05 & 4.56061 \\
\hline$u$ & 4 & 5 & 6 & 7 \\
\hline$y_{0}(u ; b)$ & 16.1795 & -33.3598 & 92.6721 & -220.969 \\
\hline$y_{1}(u ; b)$ & -8.96415 & 25.5918 & -60.2312 & 155.915 \\
\hline$u$ & 8 & 9 & 10 & 11 \\
\hline$y_{0}(u ; b)$ & 568.161 & -1406.46 & 3548.14 & -8866.63 \\
\hline$y_{1}(u ; b)$ & -384.679 & 972.075 & -2427.14 & 6096.64 \\
\hline$u$ & 12 & 13 & 14 & 15 \\
\hline$y_{0}(u ; b)$ & 22262.4 & -55764.5 & 139848 & -350509 \\
\hline$y_{1}(u ; b)$ & -15268.1 & 38293.9 & -95973.1 & 240620 \\
\hline
\end{tabular}

TABLE 3: Numerical results of $\alpha_{i}$ for $\theta=0.3$.

\begin{tabular}{lcccrr}
\hline$b$ & 15 & 16 & 17 & 18 & 19 \\
\hline$\alpha_{0}$ & 0.07130 & 0.05689 & 0.04539 & 0.03621 & 0.02889 \\
$\alpha_{1}$ & 0.10387 & 0.08287 & 0.06612 & 0.05275 & 0.04209 \\
\hline
\end{tabular}

TABLE 4: Numerical results of $\alpha_{i}$ for $b=15$.

\begin{tabular}{lccccc}
\hline$\theta$ & 0.1 & 0.15 & 0.2 & 0.25 & 0.3 \\
\hline$\alpha_{0}$ & 0.19716 & 0.16288 & 0.12968 & 0.09878 & 0.07130 \\
$\alpha_{1}$ & 0.27582 & 0.22961 & 0.18453 & 0.14212 & 0.10387 \\
\hline
\end{tabular}

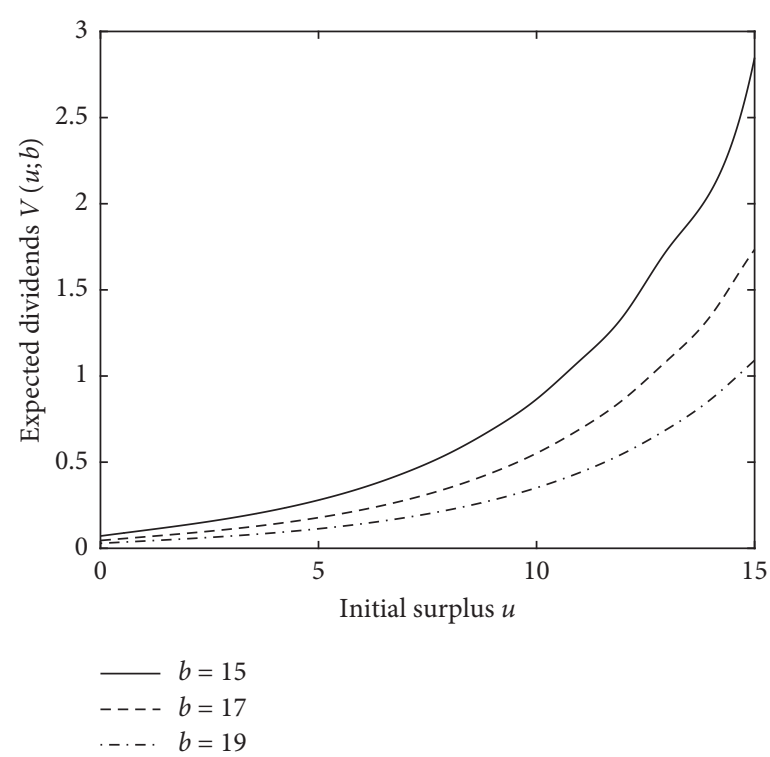

(a)

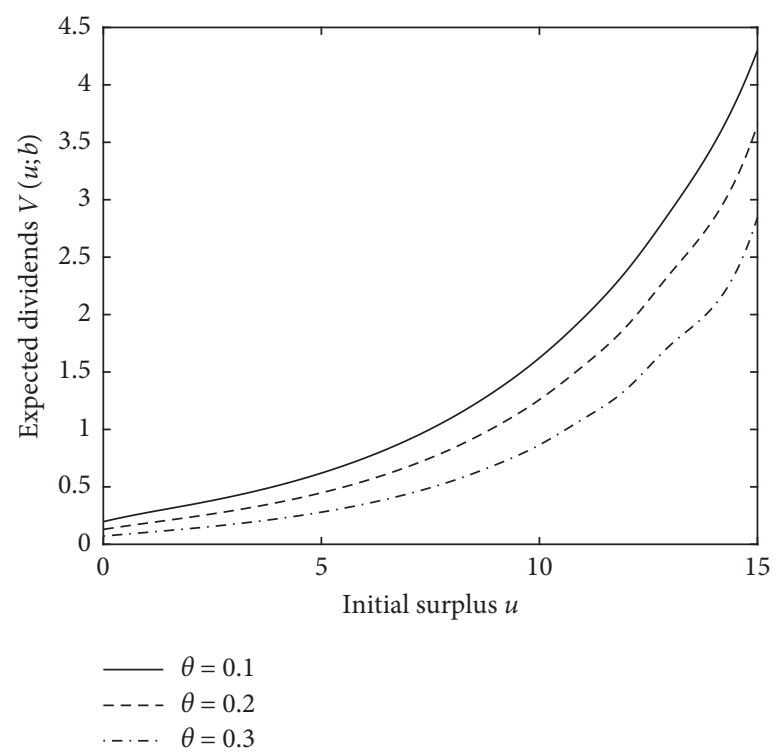

(b)

Figure 1: Impact of $\theta$ and $b$ for $V(u ; b)$. (a) Numerical results for $V(u ; b)$ when $\theta=0.3$. (b) Numerical results for $V(u ; b)$ when $b=15$. 


$$
\begin{aligned}
y_{0}(u ; b)= & 0.36125 \times(-2.50685)^{u}+0.77878 \\
& \times 1.25337^{u}-0.14003 \times 0.50348^{u}, \\
y_{1}(u ; b)= & -0.24797 \times(-2.50685)^{u}+0.33810 \\
& \times 1.25337^{u}-0.09014 \times 0.50348^{u} .
\end{aligned}
$$

Then, we obtain the values of $y_{j}(u ; b)$, see Table 2 .

To finish the calculation of $V(u ; b)$, we solve the system of linear equations (32) satisfied by $\alpha_{i}$, see Tables 3 and 4 , respectively.

We depict $V(u ; b)$ in Figure 1 , and it is not surprising that $V(u ; b)$ is an increasing function of $u$. When $\theta=0.3$ is fixed, the first part of Figure 1 shows that a larger value of $b$ corresponds to a smaller amount of dividend payments. On the contrary, for a fixed $b=15$, the second part of Figure 1 illustrates that a smaller $\theta$ leads to a larger number of dividend payments.

\section{Concluding Remarks}

In this paper, we consider the compound binomial model with random income and a constant dividend barrier. Furthermore, we analyze the model with a general premium rate $c$. Using the roots of a generalization of Lundberg's fundamental equation and the general theory on difference equations, we derive an explicit expression for the expected present value of dividend payments up to the time of ruin. In particular, a numerical example is provided to show that the formulae are readily programmable in practice. From the numerical example given above, we can see that the barrier level has a negative effect on the total expected present value of dividends.

\section{Data Availability}

The data used to support the findings of this study are included within the article.

\section{Conflicts of Interest}

The authors declare that they have no conflicts of interest.

\section{Authors' Contributions}

All authors have equal contributions. All authors have read and approved the final manuscript.

\section{Acknowledgments}

This research was supported by the Ministry of Education of Humanities and Social Science Project (20YJA910001), Foundation of Educational Department (W201783664), and Science and Technology Department (20180550196) of Liaoning Province.

\section{References}

[1] S. Li, Y. Lu, and J. Garrido, "A review of discrete-time risk models," Revista de la Real Academia de Ciencias Exactas,
Fisicas y Naturales. Serie A. Matematicas, vol. 103, no. 2, pp. 321-337, 2009.

[2] H. Liu and Z. Bao, "On a discrete-time risk model with general income and time-dependent claims," Journal of Computational and Applied Mathematics, vol. 260, pp. 470-481, 2014.

[3] J. Tan and X. Yang, "The compound binomial model with randomized decisions on paying dividends," Insurance: Mathematics and Economics, vol. 39, no. 1, pp. 1-18, 2006.

[4] D. Landriault, "Randomized dividends in the compound binomial model with a general premium rate," Scandinavian Actuarial Journal, vol. 2008, no. 1, pp. 1-15, 2008.

[5] J.-h. Xie and W. Zou, "Expected present value of total dividends in a delayed claims risk model under stochastic interest rates," Insurance: Mathematics and Economics, vol. 46, no. 2, pp. 415-422, 2010.

[6] X. Wu and $\mathrm{S}$. $\mathrm{Li}$, "On a discrete time risk model with timedelayed claims and a constant dividend barrier," Insurance Markets and Companies: Analyses and Actuarial Computations, vol. 3, no. 1, pp. 50-57, 2012.

[7] K. C. Yuen, M. Chen, and K. P. Wat, "On the expected penalty functions in a discrete semi-Markov risk model with randomized dividends," Journal of Computational and Applied Mathematics, vol. 311, pp. 239-251, 2017.

[8] L. Zhang and H. Liu, "On a discrete-time risk model with time-dependent claims and impulsive dividend payments," Scandinavian Actuarial Journal, vol. 2020, no. 8, pp. 1-19, 2020.

[9] A. V. Boikov, "The cramer--lundberg model with stochastic premium process," Theory of Probability \& Its Applications, vol. 47, no. 3, pp. 489-493, 2003.

[10] H. Yang and Z. Zhang, "On a class of renewal risk model with random income," Applied Stochastic Models in Business and Industry, vol. 25, no. 6, pp. 678-695, 2009.

[11] C. Labbé and K. P. Sendova, "The expected discounted penalty function under a risk model with stochastic income," Applied Mathematics and Computation, vol. 215, no. 5, pp. 1852-1867, 2009.

[12] I. Karnaukh, "Risk process with stochastic income and twostep premium rate," Applied Mathematics and Computation, vol. 217, no. 2, pp. 775-781, 2010.

[13] Z. Bao and H. Liu, "The compound binomial risk model with delayed claims and random income," Mathematical and Computer Modelling, vol. 55, no. 3-4, pp. 1315-1323, 2012.

[14] J. Zhou, X. Mo, H. Ou, and X. Yang, "Expected present value of total dividends in the compound binomial model with delayed claims and random income," Acta Mathematica Scientia, vol. 33, no. 6, pp. 1639-1651, 2013.

[15] W. G. Kelley and A. C. Peterson, Difference Equations: An Introduction with Applications, Academic Press, New York, NY, USA, 1 st edition, 2001.

[16] Z. H. Bao and Y. Liu, "A discrete-time ruin model with dependence between interclaim arrivals and claim sizes," $A d$ vances in Difference Equations, vol. 2016, no. 188, pp. 1-14, 2016.

[17] S. Li, "On a class of discrete time renewal risk models," Scandinavian Actuarial Journal, vol. 2005, no. 4, pp. 241-260, 2005. 